\title{
From General Relativity to A Simple-Harmonically Oscillating Universe, and Vice-Versa: A Review
}

\author{
Carmine Cataldo ${ }^{1}$ \\ ${ }^{1}$ Independent Researcher, PhD in Mechanical Engineering, Battipaglia (Salerno), Italy \\ Correspondence: Carmine Cataldo, Battipaglia (Salerno), Italy, E-mail: catcataldo@hotmail.it
}

Received: December 5, 2016

Accepted: January 1, 2017

Online Published: January 19, 2017

doi:10.5539/apr.v9n1p86

URL: http://dx.doi.org/10.5539/apr.v9n1p86

\begin{abstract}
In this paper two different lines of reasoning are followed in order to discuss a Universe that belongs to the so-called oscillatory class. In the first section, we start from the general writing of the first Friedmann - Lemaitre equation. Taking into account mass - energy equivalence, the so-called fluid equation is immediately deduced, with the usual hypotheses of homogeneity and isotropy, once identified the evolution of the Universe with an isentropic process. Considering equal to zero the curvature parameter, and carrying out an opportune position concerning the so-called cosmological constant, we obtain an oscillating class, to which a simple-harmonically oscillating Universe evidently belongs. In the second section, we start from a simple-harmonically oscillating Universe, hypothesized globally flat and characterized by at least a further spatial dimension. Once defined the density, taking into account a global symmetry elsewhere postulated, we carry out a simple but noteworthy position concerning the gravitational constant. Then, once established the dependence between pressure and density, we deduce, by means of simple mathematical passages, the equations of Friedmann - Lemaitre, without using Einstein's Relativity.
\end{abstract}

Keywords: Friedmann-Lemaître Equations, Oscillating Universe, Cosmological Constant, Extra Dimensions

\section{A Direct Line of Reasoning}

For a uniform Universe, with the usual hypotheses of homogeneity and isotropy, we can write the first Friedmann - Lemaitre equation (Friedmann, 1922) as follows:

$$
\left(\frac{d R}{d t}\right)^{2}=\frac{1}{3}\left(8 \pi G \rho+\lambda c^{2}\right) R^{2}-k c^{2}
$$

$R$ represents the scale factor, $G$ the gravitational constant, $\rho$ the density, $\lambda$ the so-called cosmological constant, $k$ the curvature parameter, whose value depends on the hypothesized geometry, and $c$ the speed of light.

As well known, if we denote with $E$ the energy, with $T$ the thermodynamic temperature, with $S$ the entropy, with $p$ the pressure, and with $V$ the volume, we can write the following:

$$
d E=T d S-p d V
$$

If we identify the evolution of the Universe with an isentropic process, from the previous relation we obtain:

$$
d E+p d V=0
$$

According to mass-energy equivalence (Einstein, 1916), we have:

$$
E=M c^{2}
$$

Obviously, we can write:

$$
\frac{d M}{d t}=\frac{d}{d t}(\rho V)=V \frac{d \rho}{d t}+\rho \frac{d V}{d t}
$$

Taking into account the foregoing relation and mass-energy equivalence, from (3) we immediately obtain:

$$
c^{2} V \frac{d \rho}{d t}+c^{2} \rho \frac{d V}{d t}+p \frac{d V}{d t}=0
$$




$$
\begin{aligned}
& V \frac{d \rho}{d t}+\rho \frac{d V}{d t}+\frac{p}{c^{2}} \frac{d V}{d t}=0 \\
& V \frac{d \rho}{d t}+\left(\rho+\frac{p}{c^{2}}\right) \frac{d V}{d t}=0
\end{aligned}
$$

From the previous relation, we may immediately deduce the so-called fluid equation:

$$
\dot{\rho}=\frac{d \rho}{d t}=-\frac{1}{V} \frac{d V}{d t}\left(\rho+\frac{p}{c^{2}}\right)=-\frac{3}{R} \frac{d R}{d t}\left(\rho+\frac{p}{c^{2}}\right)=-3 \frac{\dot{R}}{R}\left(\rho+\frac{p}{c^{2}}\right)
$$

According to Zeldovich (Zeldovich, 1961), the relation between pressure and density can be expressed as follows:

$$
p=(v-1) \rho c^{2}
$$

The value of $v$, hypothesized as being constant, depends on the fluid, evidently postulated as being ideal, with which we identify the Universe.

From the previous equation, taking into account (8), we can immediately write:

$$
\begin{gathered}
V \frac{d \rho}{d t}+v \rho \frac{d V}{d t}=0 \\
R^{3} \frac{d \rho}{d t}+3 v \rho R^{2} \frac{d R}{d t}=0 \\
\frac{d \rho}{\rho}=-3 v \frac{d R}{R}
\end{gathered}
$$

As a consequence, if we denote with $C$ the constant of integration, we can immediately write:

$$
\begin{gathered}
\rho=C R^{-3 v} \\
\rho R^{3 v}=C
\end{gathered}
$$

Equation (1) can be written as follows:

$$
\left(\frac{d R}{d t}\right)^{2}=\frac{8 \pi G \rho R^{3 v}}{3} R^{2-3 v}+\frac{1}{3} \lambda c^{2} R^{2}-k c^{2}
$$

We can now define the following new constant (Harrison, 1967):

$$
C_{v}=\frac{8 \pi G \rho R^{3 v}}{3}=\frac{8 \pi G C}{3}
$$

Finally, by substituting the previous identity into (16), we obtain:

$$
\dot{R}^{2}=C_{v} R^{2-3 v}+\frac{1}{3} \lambda c^{2} R^{2}-k c^{2}
$$

If we denote with $\omega$ the pulsation of the Universe we want to describe, we can carry out the following position:

$$
\lambda=-3\left(\frac{\omega}{c}\right)^{2}
$$

If we set the curvature parameter equal to zero, by substituting (19) into (18), we finally obtain:

$$
\dot{R}^{2}=C_{v} R^{2-3 v}-\omega^{2} R^{2}
$$

From the previous equation, we can deduce as follows:

$$
\begin{gathered}
\dot{R}^{2}=C_{v} R^{2-3 v}\left(1-\frac{\omega^{2} R^{3 v}}{C_{v}}\right) \\
\frac{d R}{d t}=\sqrt{C_{v}} R^{1-\frac{3}{2} \nu} \sqrt{1-\left(\frac{\omega R^{\frac{3}{2}} v}{\sqrt{C_{v}}}\right)^{2}}
\end{gathered}
$$




$$
\begin{gathered}
\frac{1}{\sqrt{C_{v}} R^{1-\frac{3}{2} v}} \frac{d R}{\sqrt{1-\left(\frac{\omega R^{\frac{3}{2}} v}{\sqrt{C_{v}}}\right)^{2}}}=d t \\
\frac{2}{3 v \omega} \frac{d\left(\frac{\omega R^{\frac{3}{2} \nu}}{\sqrt{C_{v}}}\right)}{\sqrt{1-\left(\frac{\omega R^{\frac{3}{2}} v}{\sqrt{C_{v}}}\right)^{2}}}=d t
\end{gathered}
$$

If we impose that the radius of curvature assumes a null value when $t=0$, from the prior equation we can deduce:

$$
\begin{gathered}
\sin ^{-1}\left(\frac{\omega R^{\frac{3}{2} v}}{\sqrt{C_{v}}}\right)=\frac{3}{2} v \omega t \\
R^{3 v}=\frac{C_{v}}{\omega^{2}} \sin ^{2}\left(\frac{3}{2} v \omega t\right)=\frac{C_{v}}{2 \omega^{2}}[1-\cos (3 v \omega t)] \\
R=\left(\frac{C_{v}}{2 \omega^{2}}\right)^{\frac{1}{3 v}}[1-\cos (3 v \omega t)]^{\frac{1}{3 v}}
\end{gathered}
$$

From (17) and (27), we easily obtain:

$$
\rho=\frac{3}{8 \pi G} \frac{C_{v}}{R^{3 v}}=\frac{3 \omega^{2}}{4 \pi G} \frac{1}{1-\cos (3 v \omega t)}
$$

By taking into account (19) we can write the foregoing equation as follows:

$$
\rho=-\frac{\lambda c^{2}}{4 \pi G} \frac{1}{1-\cos (3 v \omega t)}
$$

If we set $v$ equal to $1 / 3$, from (27) we obtain

$$
R=\frac{C_{1 / 3}}{2 \omega^{2}}[1-\cos (\omega t)]
$$

In other terms, we have found a simple-harmonically oscillating universe, characterized by a variable density whose value, taking into account (29), is provided by the following relation:

$$
\rho=-\frac{\lambda c^{2}}{4 \pi G} \frac{1}{1-\cos (\omega t)}
$$

Obviously, if we denote with $A$ the amplitude of the motion, we can write:

$$
\begin{gathered}
A=\frac{C_{1 / 3}}{2 \omega^{2}} \\
0 \leq R \leq 2 A=\frac{C_{1 / 3}}{\omega^{2}}
\end{gathered}
$$

Finally, denoting with $R_{m}$ the mean radius, we obtain:

$$
\begin{gathered}
\omega t=\frac{\pi}{2} \rightarrow R=A=R_{m} \\
R=R_{m}[1-\cos (\omega t)] \\
\omega t=\frac{\pi}{2} \rightarrow \rho_{m}=\rho\left(R_{m}\right)=-\frac{\lambda c^{2}}{4 \pi G}
\end{gathered}
$$

\section{An Alternative Deduction of the Friedmann - Lemaître Equations}

We now want to follow an inverse line of reasoning. In other terms, we start from postulating a Universe, homogeneous and isotropic, whose evolution is described by (35). We can evidently write the following:

$$
R=R_{m}(1-\cos \alpha)
$$




$$
\begin{gathered}
\alpha=\omega t=\frac{c t}{R_{m}} \\
\cos \alpha=1-\frac{R}{R_{m}} \\
\dot{R}=\frac{d R}{d t}=c \sin \alpha \\
\ddot{R}=\frac{d \dot{R}}{d t}=c \omega \cos \alpha=\frac{c^{2}}{R_{m}}\left(1-\frac{R}{R_{m}}\right)
\end{gathered}
$$

By virtue of (37) and (40), we can write the so-called Hubble parameter (Hubble, 1929), denoted by $H$, as follows:

$$
H=\frac{\dot{R}}{R}=\frac{c}{R_{m}} \frac{2 \sin \left(\frac{\alpha}{2}\right) \cos \left(\frac{\alpha}{2}\right)}{2 \sin ^{2}\left(\frac{\alpha}{2}\right)}=\frac{c}{R_{m}} \frac{1}{\tan \left(\frac{c t}{2 R_{m}}\right)}
$$

Obviously, when $t=0$ we have a singularity. This and other problems related to the Universes belonging to the oscillatory class (Turok \& Steinhardt, 2005) may be solved by assigning a different meaning to the metric variation of distances. Although the topic will not be herein considered, we may state that the discussion would have started by postulating a Universe actually static, characterized, net of symmetry, by at least five dimensions.

We hypothesize a closed Universe, globally flat, characterized by at least a further spatial dimension (Cataldo, 2016a). Such a Universe, identifiable with a four-dimensional ball, is described by the following inequality:

$$
x_{1}^{2}+x_{2}^{2}+x_{3}^{2}+x_{4}^{2} \leq R^{2}
$$

However, the Universe we are allowed to perceive, when we are at rest, can be assimilated to the hyper-surface described by the underlying equation:

$$
x_{1}^{2}+x_{2}^{2}+x_{3}^{2}+x_{4}^{2}=R^{2}
$$

Consequently, we can write the hyper-volume of the Universe in its entirety as follows:

$$
V_{4}=\frac{1}{2} \pi^{2} R^{4}
$$

As for the volume of the curved space we believe to live in, we can evidently write:

$$
\partial V_{4}=\frac{d V_{4}}{d R}=2 \pi^{2} R^{3}
$$

Obviously, we are not allowed to perceive such a Universe in its entirety. Net of the symmetry, what we perceive as a material point at rest may be nothing but one of the intersections between a material segment, that crosses the center of the 4-ball defined by (43), and the hyper-surface defined by (44) (Cataldo, 2016b). If we denote, as usual, with $a(t)$ the scale factor, and with $\chi$ the angular distance, as perceived by an ideal observer placed right at the center of the 4-ball defined by (43), between two points, one of which is taken as origin, belonging to the hyper-surface defined by (44), the curved space we perceive can be effectively described, with obvious meaning of symbols and signs, by means of the well-known Robertson-Walker metric:

$$
\begin{gathered}
a(t)=R \\
r=\sin \chi \\
d s^{2}=c^{2} d t^{2}-a^{2}(t)\left[\frac{d r^{2}}{1-r^{2}}+r^{2}\left(d \theta^{2}+\sin ^{2} \theta d \varphi^{2}\right)\right]
\end{gathered}
$$

Our analysis will be carried out by considering one amongst the scenarios that arise from (43) and (44), once having set equal to zero one of the coordinates. In other terms, the Universe in its entirety is identified with a ball, and the curved space we are allowed to perceive is assimilated to a spherical surface.

Taking into account the symmetry (Cataldo, 2016c), denoting with $M$ the mass of the whole Universe, we can now define the density as follows: 


$$
\rho=\frac{M}{\frac{2}{3} \pi R^{3}}
$$

If $M_{m}$ represents the mass when $R=R_{m}$, from the prior equation we obtain:

$$
\rho_{m}=\rho\left(R_{m}\right)=\frac{M_{m}}{\frac{2}{3} \pi R_{m}^{3}}=\frac{3 \frac{c^{2}}{R_{m}^{2}}}{4 \pi \frac{c^{2} R_{m}}{2 M_{m}}}
$$

Let's carry out the underlying noteworthy position (Cataldo, 2016d):

$$
G=\frac{R_{m} c^{2}}{2 M_{m}}
$$

From the previous identity, by virtue of which we may identify $R_{m}$ with the Schwarzschild radius (Schwarzschild, 1919) of the Universe, taking into account (51), we obtain:

$$
\rho_{m}=\frac{3 \frac{c^{2}}{R_{m}^{2}}}{4 \pi G}
$$

Now, we can define the so-called cosmological constant as follows:

$$
\lambda=-\frac{3}{R_{m}^{2}}
$$

In accordance with the foregoing position, from (53) we immediately obtain what already deduced in (36).

If we identify the evolution of the Universe with an isentropic process, taking into account the relation between pressure and density, denoting with $V_{m}$ the mean volume, we can write:

$$
\begin{gathered}
\frac{d}{d t}\left(p V^{v}\right)=0 \\
\frac{d}{d t}\left(\rho V^{v}\right)=0 \\
\rho V^{v}=\rho_{m} V_{m}^{v} \\
\rho R^{3 v}=\rho_{m} R_{m}^{3 v}
\end{gathered}
$$

We have elsewhere determined the value of $v$ by speculating about the degrees of freedom that can be ascribed to whatever material point belonging to a Universe like the one we have hypothesized (Cataldo, 2016e). Actually, we could have followed further different lines of reasoning. For example, the Newtonian gravitational field produced by a generic mass $m$ can be written as follows:

$$
g=\frac{G m}{d^{2}}
$$

We can approximatively identify $d$ with the measured distance between the gravitational source and the point in correspondence of which we want to evaluate the field. Alternatively, taking into account a possible pseudo-Newtonian gravity, whose expression should obviously resemble (59), we could simply impose a linear dependence between $d$, that would no longer be identifiable with the measured distance, and the radius of the Universe (Cataldo, 2016f). Hence, for a generic source $m$, once fixed the angular distance, we may write:

$$
\begin{gathered}
d=f(R, \chi) \propto R \\
g \propto \frac{m}{R^{2}}
\end{gathered}
$$

To maintain the field constant, generalizing (61), we must write:

$$
\begin{gathered}
\frac{d}{d t}\left(\frac{M}{R^{2}}\right)=0 \\
\frac{M}{R^{2}}=\frac{M_{m}}{R_{m}^{2}}
\end{gathered}
$$




$$
\rho R=\rho_{m} R_{m}
$$

By comparing the previous equation with (58), we immediately obtain:

$$
v=\frac{1}{3}
$$

In other terms, we are considering the variations of cosmological distances as being exclusively metric: alternatively, we are postulating that the amount of space between whatever couple of points remains the same with the passing of time. As a consequence, if we assign a variable value to cosmological distances, coherently with the apparent evolution of the Universe, we also have to assign, to maintain the gravitational field constant, a variable value to the mass that produces the field itself.

Obviously, by taking into account (10), we can immediately write:

$$
p=-\frac{2}{3} \rho c^{2}
$$

From (36) and (64) we immediately deduce the following:

$$
\begin{gathered}
\rho=\frac{R_{m}}{R} \rho_{m}=\frac{3}{4 \pi G} \frac{c^{2}}{R R_{m}} \\
\frac{c^{2}}{R R_{m}}=\frac{4 \pi G}{3} \rho
\end{gathered}
$$

From (39) and (40) we easily obtain:

$$
\begin{aligned}
\dot{R}^{2}=c^{2}\left(1-\cos ^{2} \alpha\right) & =2 c^{2} \frac{R}{R_{m}}-c^{2} \frac{R^{2}}{R_{m}^{2}} \\
\dot{R}^{2}+c^{2} \frac{R^{2}}{R_{m}^{2}} & =2 c^{2} \frac{R}{R_{m}}
\end{aligned}
$$

If the radius is different from zero, considering the previous relation, by virtue of (68), we may write:

$$
\left(\frac{\dot{R}}{R}\right)^{2}+\frac{c^{2}}{R_{m}^{2}}=2 \frac{c^{2}}{R R_{m}}=\frac{8 \pi G}{3} \rho
$$

Taking into account (54), from the foregoing relation we obtain:

$$
\begin{gathered}
\left(\frac{\dot{R}}{R}\right)^{2}-\frac{\lambda c^{2}}{3}=\frac{8 \pi G}{3} \rho \\
\left(\frac{d R}{d t}\right)^{2}=\frac{1}{3}\left(8 \pi G \rho+\lambda c^{2}\right) R^{2}
\end{gathered}
$$

Obviously, the previous equation is nothing but (1) with the curvature parameter equal to zero.

Now, we may easily rearrange (69) as follows:

$$
\dot{R}^{2}=2 R \frac{c^{2}}{R_{m}}\left(1-\frac{R}{R_{m}}\right)+c^{2} \frac{R^{2}}{R_{m}^{2}}
$$

From the foregoing equation, by virtue of (41) and (54), we can deduce:

$$
\begin{gathered}
\dot{R}^{2}=2 R \ddot{R}+c^{2} \frac{R^{2}}{R_{m}^{2}}=2 R \ddot{R}-\frac{\lambda c^{2}}{3} R^{2} \\
\left(\frac{\dot{R}}{R}\right)^{2}=2 \frac{\ddot{R}}{R}-\frac{\lambda c^{2}}{3}
\end{gathered}
$$

From (72), taking into account (66), we can write the following:

$$
\left(\frac{\dot{R}}{R}\right)^{2}-\frac{\lambda c^{2}}{3}=-\frac{4 \pi G}{c^{2}} p
$$

If we multiply by two the first and second member of the previous equation, we immediately obtain: 


$$
2\left(\frac{\dot{R}}{R}\right)^{2}-\frac{2}{3} \lambda c^{2}=\left(\frac{\dot{R}}{R}\right)^{2}+\left(\frac{\dot{R}}{R}\right)^{2}-\frac{2}{3} \lambda c^{2}=-\frac{8 \pi G}{c^{2}} p
$$

From the previous relation, by taking into account (76), we obtain the second Friedmann- Lemaitre equation:

$$
2 \frac{\ddot{R}}{R}+\left(\frac{\dot{R}}{R}\right)^{2}-\lambda c^{2}=-\frac{8 \pi G}{c^{2}} p
$$

\section{References}

Cataldo, C. (2016a). Towards a New Relativity: how to Travel Faster than Light. Research \& Reviews: Journal of Pure and Applied Physics, 4(1), 7-14. Retrieved from http://www.rroij.com/open-access/towards-a-new -relativity-how-to-travel-faster-than-light-.pdf

Cataldo, C. (2016b). Faster than Light: again on the Lorentz Transformations. Applied Physics Research, 8(6), 17-24. http://dx.doi.org/10.5539/apr.v8n6p17

Cataldo, C. (2016c). Effects of a Global Symmetry on the Observation of Astronomical Objects. Applied Physics Research, 8(5), 75-80. http://dx.doi.org/10.5539/apr.v8n5p75

Cataldo, C. (2016d). A simplified model of Oscillating Universe: alternative deduction of Friedmann-Lemaitre equations with a negative cosmological constant. Research \& Reviews: J. of Pure and App. Phys., 4(2), 1-3. Retrieved from http://www.rroij.com/open-access/a-simplified-model-of-oscillating-universe-alternative -deduction-of-friedmannlematre-equations-with-a-negative-cosmological-consta-.pdf

Cataldo, C. (2016e). Further Remarks on the Oscillating Universe: an Explicative Approach. Research \& Reviews: Journal of Pure and Applied Physics, 4(3), 3-37. Retrieved from http://www.rroij.com/openaccess/further-remarks-on-the-oscillating-universe-an-explicative-approach-.pdf

Cataldo, C. (2016f). Beyond the Metrics: qualitative introduction to the Modified Gravitation. Research \& Reviews: Journal of Pure and Applied Physics, 4(2). Retrieved from http://www.rroij.com/open-access/ beyond-the-metrics-qualitative-introduction-to-the-modified-gravitation-.pdf

Einstein, A. (1916). Relativity: The Special and General Theory (translated by R. W. Lawson, 1920). Henry Holt and Company, New York. Retrieved from https://archive.org/details/cu31924011804774

Friedmann A. (1922). Über die Krümmung des Raumes. Zeitschrift für Physik, 10, 377-386. English Translation (1999) On the Curvature of Space. General Relativity and Gravitation, 31(12), 1991-2000. Retrieved from http://www.ymambrini.com/My_World/History_files/Friedman_1922.pdf

Harrison, E. R. (1967). Classification of uniform cosmological models. Monthly Notices of the Roy. Astron. Soc., 137, 69-79. Retrieved from http://paperity.org/p/39522229/classification-of-uniform -cosmological-models

Hubble E. (1929). A Relation between Distance and Radial Velocity among Extra-Galactic Nebulae. Proceedings of the National Academy of Sciences of the United States of America, 15, 168-173. Retrieved from http://www.ncbi.nlm.nih.gov/pmc/articles/PMC522427/pdf/pnas01016-0006.pdf

Schwarzschild K. (1919). Über das Gravitationsfeld eines Massenpunktes nach der Einsteinschen Theorie. Sitzungsberichte der Deutschen Akademie der Wissenschaften zu Berlin, 189-196 (On the Gravitational Field of a Mass Point according to Einstein's Theory, translated by Antoci S and Loinger A, 1999). Retrieved from http://zelmanov.ptep-online.com/papers/zj-2008-03.pdf

Turok, N., Steinhardt P. J. (2005). Beyond Inflation: A Cyclic Universe Scenario. Physica Scripta. T (117), 7685. Retrieved from http://www.damtp.cam.ac.uk/user/ngt1000/neil11.pdf

Zeldovich Y. (1961). The equation of state at ultrahigh densities and its relativistic limitations. J. Exptl. Theoret. Phys. (U.S.S.R.), 41, 1609-1615. Retrieved from http://www.jetp.ac.ru/cgi-bin/dn/e_014_05_1143.pdf

\section{Copyrights}

Copyright for this article is retained by the author(s), with first publication rights granted to the journal.

This is an open-access article distributed under the terms and conditions of the Creative Commons Attribution license (http://creativecommons.org/licenses/by/4.0/). 